\title{
The Surgical Smoke in COVID Pandemic. Known and Unknown Facts
}

\author{
Pradyumna Pan* \\ Pediatric Surgery Unit, Ashish Hospital and Research Center, India
}

*Corresponding author: Pradyumna Pan, Pediatric Surgery Unit, Ashish Hospital and Research Center, Jabalpur, Madhya Pradesh, India.

\author{
Received Date: January 16, 2021 \\ Published Date: February 15, 2021
}

\section{Opinion}

A wide variety of advice and opinion from surgical societies has resulted from the COVID-19 global pandemic. The safety of surgically induced smoke is an area of controversy. There is not adequate evidence of corona virus transmission through surgical smoke. Measures should, however, be taken to manage the possible risks.

In a short period, the corona crisis has reached gigantic proportions. There is an orchestrated interruption in the normal operation of the hospital to redirect the resources available for crisis management. COVID-19, the 2020 pandemic of the novel coronavirus, has raised questions about the possibility of transmission of viruses to operating room workers. This refers not only to the intubation and extubating of the airway during anesthesia, but also to the release into the smoke or plume of possible infectious particles. Owing to the uncertain prevalence of asymptomatic COVID-19 carriers, conducting surgeries during this pandemic poses risks to the surgical team in the absence of widespread monitoring. Aerosol development during the activity of these tissues, given the highly contagious transmissibility.

As COVID-19 has been documented in different organs of the body, aerosol production during surgery on these tissues is critically relevant. Surgical smoke is a gaseous substance associated with tissue cauterization and thermal necrosis caused by thermal equipment. It is composed of $95 \%$ water/steam and $5 \%$ blood and tissue particles, chemicals, viruses, and bacteria. The size of the particulate produced depends on the thermal system. It varies from (0.07 microns) to ( 0.31 microns). Larger particles greater than 5 microns are deposited on the walls of the upper airways and bronchus, while those smaller than 2 microns reach the bronchioles and alveoli. Most surgical masks has inadequate fitness to ensure adequate respiratory protection from surgical smoke. Normal surgical masks are capable of filtering particles 5 microns and above while a greater part of particles aerosolized in a surgical plume are less than 1 microns hence adequate filtration is also not received. With better filtering and face seal capabilities, $\mathrm{N} 95$ grade masks are designed for filter efficiency of more than $95 \%$ for 0.3 micron particles.

Laparoscopic surgery was considered to increase the risk of aerosol dispersal through pneumo- peritoneum leakage. At present there is no evidence to prove that COVID-19 is transmissible through surgical smoke. Earlier studies have, shown the existence of different viruses in surgical smoke, including Corynebacterium, human papillomavirus, poliovirus, human immunodeficiency virus and hepatitis B. The probability of disease transmission through surgical smoke does exist in humans. Viruses with structural variations have different behavior, and the presence of a virus in the surgical smoke does not inevitably imply an airborne contagion. In the absence of conclusive evidence on the safety of bioaerosols generated during surgery, a multipronged approach is necessary to protect all surgical personnel to mitigate infectious and toxic smoke transmission during surgery The following recommendation should be followed (a) small port-site incisions, (b) use of electrosurgical units at a low setting and monopolar diathermy pencils with smoke evacuators, and (c) use of filter during evacuation of pneumoperitoneum prior to specimen extraction. In addition to 
these actions, the appropriate use of PPE is recommended for the entire surgical team. Wearing eye protection, an appropriately fitted highly efficient respirator mask and disposable gear is critical to limiting contact with droplets or body fluids.

\section{Acknowledgement}

None.

\section{Conflict of Interest}

No conflict of interest. 